Cristina S. Nieves, MD

Rubiliza DC. Onofre, MD

Fortuna Corazon A. Aberin-Roldan, MD

Rene Louie C. Gutierrez, MD

Department of Otolaryngology Head and Neck Surgery East Avenue Medical Center

\section{Nasopharyngeal Tuberculosis in a Patient Presenting With Upper Airway Obstruction}

\begin{abstract}
Objective: To report a rare case of upper airway obstruction from multiple pharyngeal masses due to nasopharyngeal tuberculosis in a 22-year-old male.
\end{abstract}

\section{Methods:}

\author{
Design: Case Report \\ Setting: Tertiary Government Hospital \\ Patient: One
}

Result: A 22-year-old Filipino male with upper airway obstruction from multiple pharyngeal masses was diagnosed to have nasopharyngeal tuberculosis by histopathology. He improved after six months of anti-tuberculosis medications.

Conclusion: Tuberculosis should not be overlooked in the differential diagnoses of nasopharyngeal masses because of the difference in its management, and swift and adequate cure, compared to other nasopharyngeal pathologies.

Keywords: tuberculosis, nasopharynx, mycobacteria, hypopharyngeal mass, upper airway obstruction

Tuberculosis is the sixth leading cause of morbidity and mortality in the Philippines, which ranks highest in Southeast Asia for number of cases per head and ninth in the world for total number of tuberculosis cases. ${ }^{1}$ Almost two thirds of Filipinos have tuberculosis, and up to five million people 2 are infected yearly in our country making it a major public health concern.

Nasopharyngeal tuberculosis is a rare type of extrapulmonary tuberculosis comprising only less than $1 \%$ of tuberculosis found in the upper respiratory tract. ${ }^{3}$ The disease may present on endoscopy as a normal nasopharynx or with mucosal irregularity and ulceration, bulging of the pharyngeal wall or as a polypoid mass that may be accompanied by epistaxis, chronic cough, nasal congestion and in some cases, diplopia. ${ }^{4,5}$ We found no report of nasopharyngeal tuberculosis in the Philippine Journal of Otolaryngology Head and Neck Surgery and Philippine Journal of Internal Medicine or in a search of Herdin. Neither did we find any reports of upper airway obstruction from nasopharyngeal tuberculosis in a PubMed search using the key words "Nasopharyngeal Tuberculosis" or "Upper Airway Tract Tuberculosis". 


\section{CASE REPORTS}

We describe an unusual case of a patient with nasopharyngeal tuberculosis presenting with upper airway obstruction.

\section{CASE REPORT}

A twenty-two-year-old male with a four-year history of gradually enlarging bilateral neck masses, hyponasal speech, progressive dyspnea and dysphagia presented with impending upper airway obstruction. The patient was initially seen with sternal and intercostal retractions, stridor and tachypnea. He denied any epistaxis, ear symptoms or chronic cough, had an eleven-pack-year smoking history and lived with his father who had been previously diagnosed with and inadequately

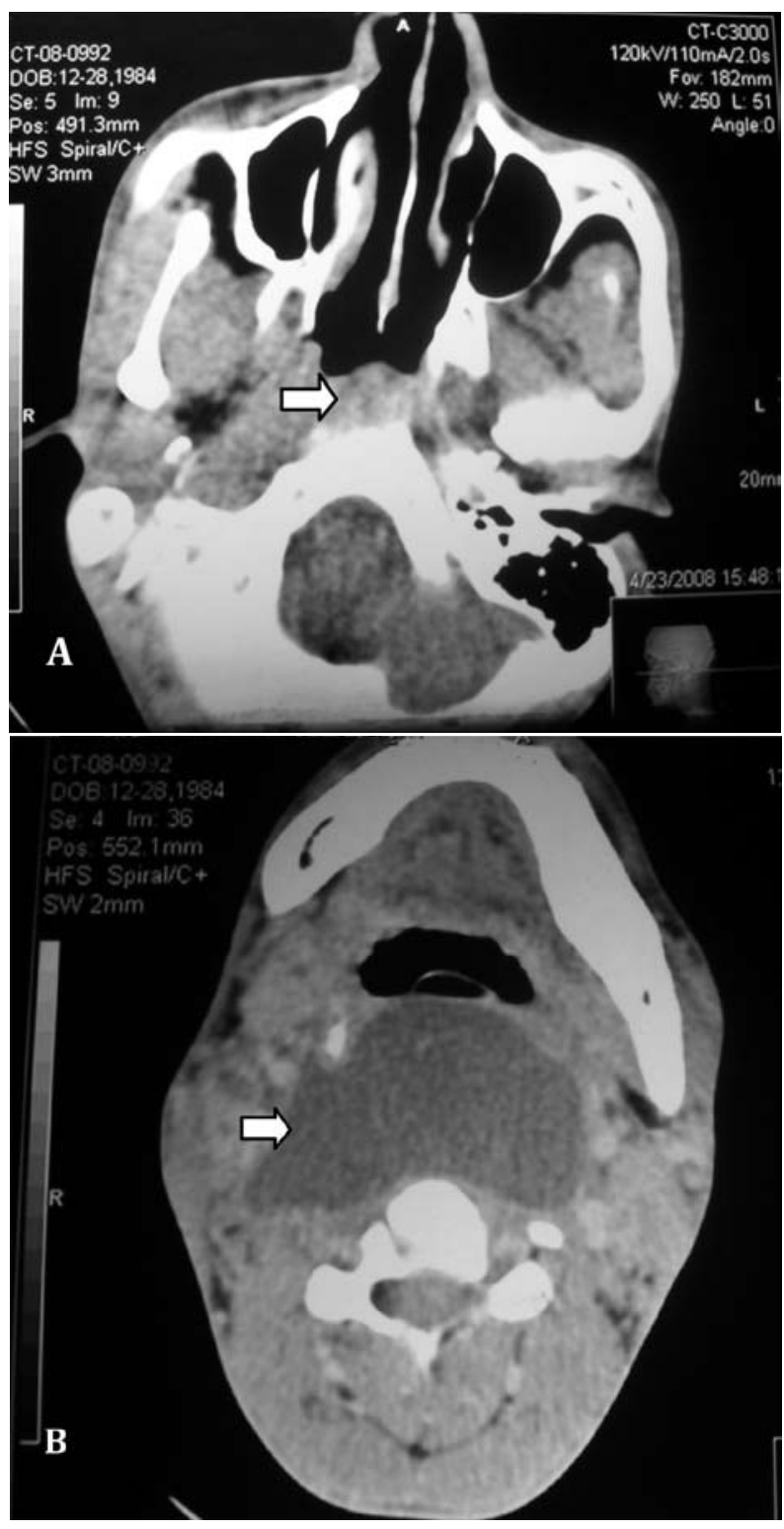

Figure 1. Computed Tomography Scan of the Pharynx. (A) Plain axial CT scan showing mucosal thickening of the nasopharynx and (B) a retropharyngeal cystic mass. treated for pulmonary tuberculosis.

Initial endoscopy revealed midline masses bulging from the midline posterior nasopharyngeal and hypopharyngeal walls, approximately $2 \mathrm{~cm} \times 1 \mathrm{~cm}$ and $2 \mathrm{~cm} \times 2 \mathrm{~cm}$ in size, respectively. There were wellcircumscribed, matted, doughy, movable, bilateral level II and III cervical lymph nodes measuring $5 \mathrm{~cm} \times 4 \mathrm{~cm} \times 1 \mathrm{~cm}$ on the right and $2.7 \mathrm{~cm} \times$ $3.6 \mathrm{~cm} \times 1 \mathrm{~cm}$ on the left.

Hematology showed only slight anemia with a normal white blood cell count. Chest radiographs revealed homogenous opacification of the left upper lung field and fibrous infiltrates in both lower lung fields. Computed tomography of the paranasal sinuses and neck showed a retropharyngeal mass, mucosal thickening in the nasopharynx, and multiple cervical lymphadenopathies. (Figure 1)

Progression of dyspnea necessitated a tracheotomy and hypopharyngeal aspiration-biopsy that yielded $14 \mathrm{~mL}$ of serosanguinous fluid with resolution of dyspnea and dysphagia.

A nasopharyngeal biopsy revealed chronic granulomatous inflammation with caseation necrosis and Langhans giant cells compatible with a tuberculous etiology of the nasopharyngeal mass. Cytologic examination of the hypopharyngeal aspirate showed chronic inflammation with mild dysplasia. Acid fast staining was negative. Sputum culture and sensitivity with acid fast staining showed no growth and no acid fast bacilli. Purified protein derivative testing was positive with fifteen millimetres skin induration.

The nasopharyngeal and hypopharyngeal masses and lymphadenopathies resolved with a six-month regimen of antituberculosis medications consisting of isoniazid, rifampicin, pyrazinamide and ethambutol for two months followed by isoniazid and rifampicin for four months. (Figure 2)

\section{DISCUSSION}

Nasopharyngeal tuberculosis is an uncommon entity and comprises only $0.12 \%$ of all tuberculosis. ${ }^{3}$ Other upper respiratory tract sites that may have tuberculous involvement are the tonsils, hypopharynx, tongue, soft palate and the larynx. ${ }^{6}$ Generally, the upper respiratory tract is thought to be resistant to tuberculosis because saliva has an inhibitory effect on tubercle bacilli. The thickness of the protective epithelial covering and antagonism of the striated musculature to bacteria also defend against tuberculosis whose pathophysiology usually requires a disruption of local mucosa leading to implantation from other foci in the body (e.g. inoculation from sputum). This may explain the localization of ulcers in the posterior part of the roof and the posterior wall of the nasopharynx. Although difficult to confirm, hematogenous spread from pulmonary tuberculosis is also considered as another means of infection, as is activation of dormant acid fast bacilli 


\section{CASE REPORTS}

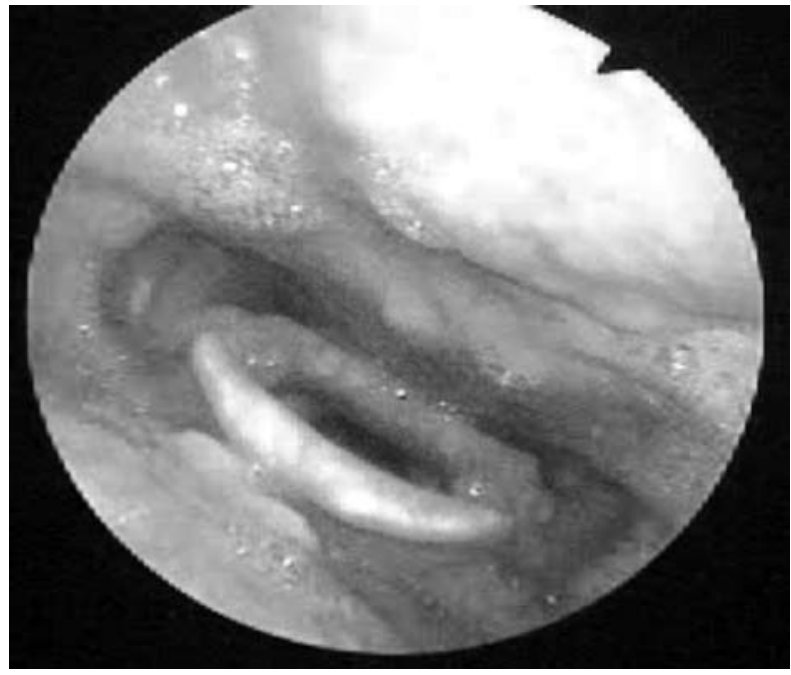

Figure 2. Hypopharynx after 6 Months of Anti-tuberculous Medications. Flexible laryngoscopy of the patient showing resolution of the retropharyngeal mass.

in adenoids during childhood. ${ }^{5}$ The disease may occur as a primary infection, without involvement of any other system, or as a secondary infection, with simultaneous pulmonary involvement as in our case.

The most common symptom of nasopharyngeal involvement is cervical lymphadenopathy, comprising about 70\%.7 Other symptoms include chronic cough, epistaxis, nasal obstruction, tinnitus, hearing loss, otitis media and even cranial nerve involvement like diplopia. ${ }^{5}$ Varying clinical presentations make diagnosis difficult. In our case the patient did present with a nasopharyngeal mass and cervical lymphadenopathy, but he also experienced upper airway obstruction due to a hypopharyngeal mass, probably resulting from retropharyngeal lymph node involvement.

The common differential diagnoses include nasopharyngeal carcinoma, Wegener's granulomatous, sarcoidosis, syphilis, leprosy, lymphoma and fungal infection. ${ }^{9}$ The diagnosis of nasopharyngeal tuberculosis is best done by biopsy to differentiate it from other nasopharyngeal masses. Histopathologic findings in tuberculosis usually include granulomatous inflammation composed of epitheloid cells and Langhan's giant cells with or without caseation. Demonstration of acid fast bacilli or a positive culture for Mycobacterium tuberculosis gives a definite diagnosis, but few specimens demonstrate acid fast bacilli with the majority yielding negative results. ${ }^{10}$

Diagnostic imaging is inaccurate in confirming the diagnosis. The gold standard for the diagnosis of nasopharyngeal tuberculosis remains to be tissue biopsy. Treatment for extrapulmonary and pulmonary tuberculosis is the same unless organisms are known and strongly suspected to be resistant to the first line drugs, or if the patient is diagnosed with tuberculous meningitis. ${ }^{2}$ In our patient, a sixmonth regimen consisting of isoniazid, rifampicin, pyrazinamide and ethambutol for two months as the initial phase followed by isoniazid and rifampicin for four months as the continuation phase was advocated.

The resolution of the nasopharyngeal mass and cervical lymphadenopathies after completion of the anti-tuberculous medications, further confirms our diagnosis of nasopharyngeal tuberculosis. Tuberculosis should not be overlooked in the differential diagnoses of nasopharyngeal masses because of the difference in its management, and swift and adequate cure, compared to other nasopharyngeal pathologies.

\section{REFERENCES}

1. World Health Organization. Global Tuberculosis Control: Surveillance, Planning Financing. Geneva: WHO 2005. [cited 2009 Jan 20]; Available from http://apps.who. int/globalatlas/predefinedReports/TB/PDF_Files/phl.pdf

2. Task Force on Tuberculosis, Philippine Practice Guidelines Group in Infectious Diseases. Pulmonary Tuberculosis: Clinical Practice Guidelines. PPGG-ID Philippine Society for Microbiology and Infectious Diseases, Inc., Philippine College of Chest Physicians and Philippine Coalition Against Tuberculosis. 2006

3. Rohwedder JJ. Upper respiratory tract tuberculosis: 16 cases in a general hospital Ann Intern Med 1974; 80:708-13.

4. King AD, Ahuja AT, Gary MK, Van Hasselt $C A$, Chan AB. MR imaging features of nasopharyngeal tuberculosis: report of three cases and literature review. $A m \mathrm{~J}$ Neuroradiol 2003; 24:279-82. [cited 2009 Jan 20]; Available from http://www.ajnr.org/ cgi/reprint/24/2/279.pdf

5. Sithinamsuwan $P$, Sakulsaengprapha $A$, Chinvarun $Y$. Nasopharyngeal tuberculosis: a case report presenting with diplopia. JMed Assoc Thai 2005; 88:1442-6. [cited $2009 \mathrm{Jan}$ 20]; Available from http://www.mat.or.th/journal/files/Vol88_No10_1442.pdf

6. Sierra C, Fortun J, Barros C, Melcon E, Condes E, Cobo J, et al. Extra-laryngeal Head And Neck Tuberculosis. Clin Microbiol Infect 2000; 6:644-48. [cited 2009 Jan 20]; Available from http://www3.interscience.wiley.com/cgi-bin/fulltext/120711198/PDFSTART

7. Waldron J, Van Hasselt CA, Skinner DW, Arnold M: tuberculosis of the nasopharynx: clinicopathological features. Clin Otolaryngol 1992; 17:57-9.

8. Harrison NK, Knight RK. Tuberculosis of the nasopharynx misdiagnosed as Wegener's granulomatous. Thorax 1986; 41:219-20. [cited 2009 Jan 20]; Available from http:// www.pubmedcentral.nih.gov/picrender.fcgi?artid=460299\&blobtype=pdf

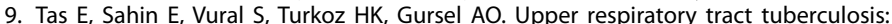
our experience of three cases and review of article: The Internet Journal of Otorhinolaryngology 2007;6:1. [cited 2009 Jan 20]; Available from http://www.ispub. com/journal/the_internet_journal_of_otorhinolaryngology/volume_6_number_ 1 18/article_printable/upper_respiratory_tract_tuberculosis_our_experience_of three_cases_and_review_of_article.html

10.Madhuri, Chandra Mohan, Sharma ML. Posterior oropharyngeal wall tuberculosis. Indian J Otolaryngol Head and Neck Surg 2002; 54:152-3. [cited 2009 Jan 20]; Available from http://medind.nic.in/ibd/t02/i2/ibdt02i2p152o.pdf 